\title{
Bild der Wissenschaft
}

Neuere Sammelbände zum Thema Visualisierung und Öffentlichkeit

Cornelius Borck

Nikolow, Sybilla/Schirrmacher, Arne, Hg., 2007. Wissenschaft und Öffentlichkeit als Ressourcen füreinander. Studien zur Wissenschaftsgeschichte im 20. Jahrhundert. Frankfurt a. M.: Campus, brosch. 370 S., $39.90 €$, ISBN-13: 978-3593384894.

Stahnisch, Frank/Bauer, Heijko, Hg., 2007. Bild und Gestalt. Wie formen Medienpraktiken das Wissen in Medizin und Humanwissenschaften? Hamburg: LIT Verlag, geb. ii+302 S., $34.90 €$, ISBN-13: 9783825889548.

Shteir, Ann B./Lightman, Bernard, Hg., 2006. Figuring It Out. Science, Gender, and Visual Culture. Hanover, N.H., u.a.: Dartmouth College Press u.a., geb. XXX+386 S., $54.99 €$, ISBN-13: 978-584656029.

Hüppauf, Bernd/Weingart, Peter, Hg., 2008. Science Images and Popular Images of Sciences. New York: Routledge, geb. 333 S., $79.99 €$, ISBN-13: 978-0415383813.

Hüppauf, Bernd/Weingart, Peter, Hg., 2009. Frosch und Frankenstein. Bilder als Medium der Popularisierung von Wissenschaft. Bielefeld: Transcript, brosch. 459 S., $29.80 €$, ISBN-13: 978-3899428926.

Gall, Alexander, Hg., 2007. Konstruieren, Kommunizieren, Präsentieren. Bilder von Wissenschaft und Technik. Göttingen: Wallstein, geb. 475 S., $28 €$, ISBN-13: 978-3835301801. 
Elkins, James, Hg., 2008. Visual Literacy. New York [u. a.]: Routledge, geb. viii+217 S., $73.99 €$, ISBN-13: 978-0415958103.

Pauwels, Luc, Hg., 2006. Visual Cultures of Science. Rethinking Representational Practices in Knowledge Building and Science Communication. Hanover, N.H., u.a.: Dartmouth College Press, geb. xix+299 S., 80 \$, ISBN-13: 978-1584655114.

"The innocent eye is blind."

Ernst Gombrich

Vor mittlerweile zehn Jahren hat Karin Knorr Cetina die Zeit der "Viskurse“ ausgerufen, nachdem William J. T. Mitchell und Gottfried Boehm schon einige Jahre zuvor den "pictorial" beziehungsweise "iconic turn" angekündigt hatten. In der Zwischenzeit ist aus dem einst recht einsamen Grenzbereich zwischen Wissenschaftsgeschichte, Wissenschaftssoziologie, Kultur- und Kunstwissenschaften eine Zone reger Forschungsaktivitäten geworden, was schon die Fülle der jüngst erschienenen Sammelbände belegt, von denen die hier zu besprechenden nur eine kleine Auswahl darstellen. Kaum eine Woche vergeht, in der nicht die neue Dominanz der Bilder in den Wissenschaften wie in der Gesellschaft herausgestellt wird - von der Bildpolitik in Zeiten eines neuen Fundamentalismus und visuell basierter Kriegslegitimierung ganz zu schweigen. Als sich kürzlich auch die Berlin-Brandenburgische Akademie der Wissenschaften des Themas in ihren Gegenworten annahm, sollte wohl mit dem Titel „Visualisierung oder Vision“ die Brisanz der aktuellen Konjunktur eingefangen werden - aber was für eine Vision kann dabei gemeint sein? Wenigstens vorläufig bedarf es ohne Zweifel weiterhin der Visionen, um Ziele und Perspektiven eines Felds wissenschaftsreflexiver Forschung abzustecken, die trotz brillanter Einzelstudien und abstrakter Systematisierungen nur zäh voranzukommen scheint. „Viskurse“ waren einmal der Vorausblick auf blühende akademische Landschaften, heute fragt Google: „Meinten Sie Viskose?“ Offenbar können nicht nur gut gemeinte Hochschulreformen das Gegenteil ihrer Ziele generieren, denn gerade die Vielzahl an Fallstudien, Tagungsberichten und Sammelpublikationen scheint eine gewisse Stagnation des Feldes anzuzeigen.

\section{Ordnungen der Sichtbarkeit}

Die grundlegende Einsicht der historischen Wissenschaftsforschung, dass wissenschaftliche Tatsachen nicht, reine' Entdeckungen einer vorgefertigten Wirklichkeit sind, sondern vielmehr ,Tat-Sachen', also Ergebnisse gezielten wissenschaftlichen Handelns und komplexer soziotechnischer Interventi- 
onen, diese Grundeinsicht gilt selbstverständlich ganz analog für wissenschaftliche Bilder. Und doch entpuppt sich die vermeintlich innovative Ausgangshypothese dort schnell als merkwürdig stumpf. Gilt doch für Bilder, dass sie im Unterschied zu Begriff und Theorie in der Regel nicht für die Sache selbst genommen werden. Selbst im klassischen Repräsentationsparadigma, nach dem wissenschaftliche Visualisierungen eben Abbildungen, also Widerspiegelungen einer äußeren Wirklichkeit waren, galt das Urteil ihrer Inferiorität gegenüber Wort und Theorie ihrer Materialität. Nolens volens verweisen Bilder deshalb von jeher auf ihre Herstellung, selbst wenn - wie im Fall der grafischen Methode oder der Fotografie - ihre Objektivität und anonyme Autorschaft vehement inszeniert wurden. ${ }^{1}$ Gerade die alte Skepsis gegenüber Bildern, die diese als nachrangig gegenüber der Sache selbst beziehungsweise der Theorie wertet, zeigt hier ihre Kehrseite: Wer Bilder als epistemische Formen ernst nehmen will, kämpft an zwei Fronten gleichzeitig, nämlich gegen das Repräsentationsparadigma und gegen die Klassifizierung von Bildern als vermeintlich sekundäre Illustrationen eines notwendig schon vorausgesetzten Theoretischen. Eine bloße Anwendung beziehungsweise Ausweitung der in den science studies mittlerweile so gut erprobten Methodologien auf Bilder führt vielleicht auch deshalb nur wenig über den Stand der Dinge hinaus, weil mit Lorraine Dastons und Peter Galisons inspirierender doppelter Vorgabe bereits so viel gesagt scheint, dass kaum Raum für Neues bleibt: In ihrem Aufsatz The Image of Objectivity (1992) sowie in ihrem aktuellen Buch Objectivity (2007) postulieren sie, die Kategorie der Objektivität habe nicht nur selbst eine Geschichte, sondern diese Geschichte lasse sich besonders im jeweiligen historisch spezifischen Umgang mit Visualisierungen, also anhand der Differenzierungen im Einsatz von Bildern freilegen.

Was können visual studies of science also leisten? Paradoxerweise scheint heute umso lauter auf einer Konstruktion visueller Wirklichkeiten insistiert zu werden, je stiller es um den sozialen Konstruktivismus wissenschaftlichen Wissens wird und die radikalen Positionen von einst immer mehr von einem wissenschaftlichen Realismus eingeholt werden. Was genau damit gemeint sein kann, die Virtualität oder Konventionalität von Neuro- und Nanobildern zu behaupten, worin die Eigenlogik von Bildern generell bestehen könnte und wie dazu wissenschaftliche Bilder einzuordnen wären - das zu bestimmen, fällt den Autorinnen und Autoren sämtlicher Bände schwer. Derzeit scheint ihre Kraft weniger in einer methodischen Erweiterung oder Erneuerung von Wissenschaftsgeschichte und Wissenschaftsforschung als vielmehr in der detailgenauen Analyse der vielfältigen Wirksamkeit von Visualisierungen zu liegen. Als materielle Artefakte versammeln Bilder in ihrer deiktischen Dinglichkeit so verschiedene Aspekte, dass sie zu ausgezeichneten Orten des Einsatzes bereits anderswo etablierter Methoden werden. Daraus folgt selbstverständlich nicht, dass sich nicht mit oder über Bilder 
philosophieren ließe - die Beiträge von Mitchell und Dieter Mersch liefern instruktive und lesenswerte Beispiele dafür. ${ }^{2}$ Aber von Konzeption und Aufbau her beschränken die Herausgeber ihre theoretischen Innovationen in der Regel auf systematisierende Einleitungen.

Ein Resultat der mittlerweile enorm intensivierten wissenschaftlichen Forschung auf diesem Gebiet scheint dabei so etwas wie eine neue Unsicherheit über die Beziehung zwischen Bildern und Wissen zu sein. Weder gibt es einen Weg zurück zur alten Orthodoxie der reinen und bilderlosen Theorie als Kernzone wissenschaftlichen Wissens, noch scheint die Antwort der kunsthistorischen Bildkundler ganz zu überzeugen, dass Bilder sich erst jenseits dessen erschließen, was sie darstellen. Wie ließe sich etwa Gottfried Boehms These, dass Bilder etwas zu zeigen geben, auf wissenschaftliche Abbildungen oder Visualisierungen im Forschungshandeln übertragen? Und welche Aussichten eröffnet überhaupt die Entscheidung, methodisch diesen Anschluss zu suchen?

\section{Figuren des Dritten}

Wenn Bilder in den Wissenschaften immer auch Werkzeuge der Kommunikation sind, was läge näher als angesichts der kunsthistorischen Fokussierung auf das Bildliche und der langen wissenschaftsphilosophischen Dominanz von Theorie den Wettstreit von Bild und Begriff zu öffnen und die Position eines Dritten einzuführen? Die Sammelbände erweitern die festgefahrene Opposition auf die ein oder andere Weise um solche Figuren eines Dritten: Sybilla Nikolow und Arne Schirrmacher separieren bereits im Titel Wissenschaft und Öffentlichkeit, um sie sogleich „als Ressourcen füreinander" zu bestimmen. In direkter Anknüpfung an Mitchell Ashs These differenzieren sie dabei die „gegenseitige Inanspruchnahme von Wissenschaft und Öffentlichkeit" (S. 26) in ihrer theoretisch anspruchsvollen Einleitung zu einem hierarchischen Modell gestufter Wissensvermittlung. Damit wollen sie eine nicht zuletzt wissenschaftssoziologisch valide Alternative zum eindimensionalen Konzept einer Wissenschaftspopularisierung vom Zentrum Wissenschaft in eine als Peripherie gedachte Öffentlichkeit formulieren, die gerade auch die wechselseitige Abhängigkeit von Wissenschaft und Politik in den modernen Wissensgesellschaften in den Blick zu nehmen erlaubt.

Zugleich ist ihr Band selbst das Produkt eines komplexen kollektiven Forschungsansatzes, bei dem die Autorinnen und Autoren der Fallstudien auf der Basis einer vorab erstellten „Fragematrix“ mit vierzehn (!) Punkten $\mathrm{zu}$ Autoren-Tandems für jeweils eine von fünf systematischen „Dimensionen der gegenseitigen Inanspruchnahme“ (S. 33) zusammengespannt wurden. Bei so viel systematisierender Gründlichkeit droht das Unternehmen an den eigenen Ansprüchen zu ersticken, und die Herausgeber konstatie- 
ren abschließend, dass die versammelten Fallstudien lediglich „einen ersten Überblick über verschiedene Arten von Beziehungsgeschichten“ (S. 32) geben. Mit diesem Stichwort blitzt unverhofft eine Alternative auf, wie der abstrakten Schwere des Bandes etwas selbstironische Leichtigkeit zur Seite zu stellen gewesen wäre, um den lesenswerten Einzelstudien mehr Raum zur Entfaltung zu gewähren.

Auf ähnliche Schwierigkeiten stoßen die Autoren zweier anderer Einleitungen, wenn sie zugleich theoretisch anspruchsvoll strukturieren wollen und doch die Disparatheit der Texte nicht ignorieren können. Frank Stahnisch und Heijko Bauer setzen statt auf eine ordnende Systematik emphatisch auf das Konzept der Gestalt, um mit ihm die in den Fallstudien vorgebrachten Ergebnisse in ihrem Zusammenhang zu erschließen. So reizvoll und produktiv dieser Zugriff auf den ersten Blick wirkt, kommen auch sie nicht umhin, die angesichts der Heterogenität der Studien doch recht begrenzte Reichweite des Vorschlags zu Tage treten zu lassen. Der Begriff der Gestalt verweist zu spezifisch auf eine ganz bestimmte wissenschaftliche Tradition und tritt deshalb ausgerechnet mit sachlich oder zeitlich verwandten Themen wie etwa der Physiognomik oder der Bioergographie Lewins in unauflösliche Spannungen.

Ist es da nicht konsequenter, die Konzeptualisierung von vornherein nur in der Möglichkeitsform einzuführen? Ann Shteir und Bernard Lightman schlagen diesen Weg ein, wenn sie ihren Sammelband zur Zusammenführung von Geschlechterforschung und Visualisierung einfach mit langen Fragenkatalogen erschließen, ohne den Anspruch auf eine systematische Durchdringung zu erheben. Gerade zum Thema Geschlechterpolitik, das in ihrem Sammelband das Scharnier zwischen Bild und Wissen bildet, hätte man sich allerdings, wenn schon nicht stringente Theoriebildung, so doch wenigstens stärker artikulierte Thesen gewünscht.

Einen mehr essayistischen Weg gehen Bernd Hüppauf und Peter Weingart, wenn sie in der Einleitung zu ihrem auf Englisch und Deutsch erschienenen Sammelband Frosch und Frankenstein (hinter der elegant-witzigen Alliteration verbirgt sich eine in der Gliederung leicht abgewandelte und um vier Beiträge erweiterte Version von Science Images and Popular Images of the Sciences) schlicht und einfach von "the public" beziehungsweise „der Öffentlichkeit" sprechen. Zwar stößt man auch hier auf die Sehnsucht nach Ordnung und Systematik, wenn sie mit dem schon sprachlich recht gezwungenen Doppelspiel von science images und images of science Bilder der Wissenschaft allein anhand der sozialen Zugehörigkeit und den Absichten ihrer Hersteller sauber in vier Gruppen einzuteilen hoffen - statt bei den konkreten Verwendungsweisen und Zirkulationswegen von Bildern anzusetzen, die ja spätestens dann virulent werden, wenn die Bilder die ihnen zugedachten Grenzen überschreiten. Aber jenseits des Schematismus ist in der Einleitung eine rasante Zuspitzung der von Daston und Galison vorgeschla- 
genen Periodisierung von Objektivitätskonzepten versteckt. Mit Mikro- und Röntgenfotografie als Beispielen separieren Hüppauf und Weingart nämlich bereits die Fotografie vom Paradigma der „mechanischen Objektivität“, weil mit ihr Visualisierung von einer Darstellung des Sichtbaren zur Produktion von Evidenz aus Unsichtbarem geworden sei (das ist allerdings eine These, die allein schon aufgrund der sehr viel längeren Geschichte der Mikroskopie noch zu differenzieren wäre). Diese Produktion von Evidenz sei dann mit der Digitalisierung so zugespitzt worden, dass mittlerweile gar nicht mehr zwischen Korrektur und Manipulation unterschieden werden könne, weil bei Visualisierungen im heute üblichen Modus artifizieller Natürlichkeit jedweder Endpunkt der Bildgenerierung arbiträr geworden sei. Digitale Visualisierungen, so lautet die radikale zeitdiagnostische These, die hier von Hüppauf und Weingart aufgegriffen wird, hätten längst die Differenz von Bild und Begriff erodiert und alle visuelle Evidenz zum quasi-linguistischen Zeichen transformiert, mit dem die Grenzlinie zwischen dem Realen und dem Imaginären verschwimme. Deshalb komme einer sorgfältigen Analyse der komplexen Herstellungsbedingungen visueller Bildwelten eine eminent kritische Rolle in epistemologischer Perspektive zu. Dieses Programm hat Michael Lynch allerdings schon vor mehr als zwanzig Jahren in Social Studies of Science in seinem Aufsatz "Discipline and the Material Form of Images. An Analysis of Scientific Visibility" vorgelegt. Weil seine Studie bis heute nichts an Gültigkeit eingebüßt hat, bildet sie zusammen mit anderen Nachdrucken zu Recht den Kern des von Luc Pauwels herausgegebenen Sammelbandes.

\section{Öffentlichkeit ohne Medientheorie}

Die These von der Digitalisierung als entscheidende epistemologische Zäsur in der Geschichte der Visualisierung lässt zugleich auf eine überraschende gemeinsame Leerstelle der Bände aufmerksam werden, nämlich das beinahe vollständige Fehlen von Medientheorie in den Studien. So ambitioniert kritisch alle Beitragenden auch hinsichtlich eines mehrdimensionalen Popularisierungsmodells und einer reflektierten Bildtheorie sind, so konventionell ist oft der zugrunde gelegte Medienbegriff, der jeglicher epistemologischer Brisanz entkleidet hier zumeist nur die verschiedenen Formate der Verbreitung von Information meint. Von geradezu entwaffnender Schlichtheit ist diesbezüglich der von Alexander Gall herausgegebene Band, der seine Entstehung der neuen Dauerausstellung „Foto und Film“ im Deutschen Museum verdankt. Der im Titel formulierte Dreischritt von Konstruktion, Kommunikation und Präsentation wird noch in der Einleitung zur sauberen Aufteilung der Zuständigkeiten von Wissenschaft, Medien und Museen erklärt (S. 12). Damit ist eine unterkomplexe Analyse vieler Fallstudien im Band geradezu vorprogrammiert, die in den Einzelbeiträgen dann dazu führt, dass 
das oft wunderbare und obendrein sachkundig ausgebreitete Material nicht quer über diese starren Grenzen von Wissensgenerierung, Distribution und Darstellung sprechen darf. Auch hier bestätigen natürlich Ausnahmen die Regeln. Cheryce Kramer situiert ihre Geschichte des CORBIS-Bildarchivs von Otto Bettmann nicht nur präzise im Zwischenraum von Wissenschaft, Informationstechnik und Urheberrecht, sondern sie nutzt eine Analyse der Bedingungen und Möglichkeiten von Visualisierungstechniken fast zur institutionellen Selbstreflexion, war doch ein Auslöser für ihre Fallstudie das Angebot der Firma CORBIS, auch das Bildarchiv des Deutschen Museums zu übernehmen. Und Martina Heßler zeigt sehr schön, wie erst im Zuge neuer Drucktechniken, Computerprogramme und Visualisierungsverfahren die Doppelhelix der DNA zur Ikone der Gegenwart aufsteigen konnte. Aber ist das schon eine Medientheorie wissenschaftlicher Visualisierung?

Immerhin ist damit die gesellschaftliche Wirkungskraft von Bildern angesprochen, und dazu finden sich in allen Bänden faszinierende Analysen. So beschreibt etwa Charlotte Bigg (in Hüppauf/Weingart 2008, 2009) Nadars aberwitzige Anordnungen, um im sprachlosen Medium der Porträt-Fotografie ein Interview mit Eugène Chevreul zu führen. Jonathan Smith (in Shteir/ Lightman) analysiert die unvorhergesehenen Schwierigkeiten, in die Darwin durch seinen Entschluss geriet, The Descent of Man mit Abbildungen zum Balzverhalten von Vögeln zu illustrieren (und wofür er eigens auf Vorlagen aus Brehms Thierleben zurückgriff). Zwar ließ sich bei Vögeln der Mechanismus der sexuellen Selektion besonders anschaulich machen, aber was im Bild offensichtlich war, machte die Theorie zugleich gesellschaftlich schwer akzeptabel. Sie hätte eine Kritik an den bestehenden Verhältnissen bedeutet, in denen Frauen weder politisch noch persönlich ein Wahlrecht zukam - weshalb Darwin eiligst für die zweite Auflage andere Vorlagen entwerfen ließ.

Eine ähnlich überraschende Gegenläufigkeit legt Christina Wessely (in Nikolow/Schirrmacher) frei, wobei sie mit eloquenter Präzision beweist, wie gerade eine so augenscheinlich unsinnige Idee wie die Welteislehre sich vorzüglich eignet, um subtile Verflechtungen und Wechselwirkungen zwischen akademischer Wissenschaft und kritischer Öffentlichkeit herauszuarbeiten. Im realen Diesseits des üblichen Schemas einer unidirektionalen Wissensvermittlung entlang eines Machtgefälles kann nämlich selbst noch akademische Ignoranz zum Kristallisationskeim kritischer Gegenöffentlichkeit werden. Abschließend sei immerhin angemerkt, dass Janine Marchessaults Analyse (in Shteir/Lightmann) populärer Arztserien eine gewisse Ausnahme hinsichtlich der konstatierten Vernachlässigung von Medientheorie markiert. Nur auf den ersten Blick scheint die kanadische Medienwissenschaftlerin altbekannte Stereotypien in zu Recht vergessenen Fernsehserien aufzulisten, denn dann entziffert sie diese klischeehaften Formulierungen als subtile moralische Auseinandersetzungen, die wesentlich den medizinethischen Diskurs jener Jahre prägten. 


\section{Visuelle Allgemeinbildung}

Mit der Losung „visual literacy” ruft James Elkins seine Kollegen in den Liberal Arts Colleges auf, endlich ein einführendes Curriculum zum Umgang mit Bildlichkeit neben die Great Books Courses zu stellen:

Theorizing on images, which currently takes place mainly in graduate studies in the humanities and in cognitive science, needs to move downward, toward first-year education, where it can begin to directly intervene in the ordinary education of every college student. (S. vii)

Der von ihm herausgegebene Band mit dem gleichlautenden Titel ist allerdings eher als Warnung zu nehmen, wie man dabei nicht vorgehen sollte. Das Buch stellt eine Vor-Veröffentlichung einiger Beiträge einer großen Konferenz dar, im Anschluss an bekannt lesenswerte Texte von Mitchell, Barbara Stafford und Jonathan Crary folgen geringfügig redigierte, weitgehend entbehrliche Diskussionsprotokolle und schließlich einige Texte mit pädagogisch-didaktischen Ausführungen beziehungsweise politisch-pädagogischen Reflexionen. Das alles mag noch so hingehen, wenn nicht der Band ausgesprochen lieblos gemacht worden wäre. Anstelle einer gehaltvollen Einführung beschränkt sich Elkins darauf, die Wendung visual literacy für sich zu reklamieren, obwohl er zugleich nachweist, dass sie seit über 150 Jahren in Umlauf ist. In Ermangelung geeigneter Bildvorlagen durchziehen den Band auf Veranlassung des Verlags und ohne Bezug zum Text eingeschobene, schlecht reproduzierte Abbildungen nach Vorlieben des Herausgebers - von einer bronzezeitlichen Scherbe bis zum Foto des Ehepaars Churchill. Dem in der Sache durchaus löblichen Anliegen dürfte dieses Buch somit eher einen Bärendienst erweisen.

Auch Pauwels stellt seinen Sammelband in den Dienst einer Förderung der visual literacy, wie sein konziser Eröffnungsbeitrag „A Theoretical Framework for Assessing Visual Representational Practices in Knowledge Building and Science Communcations" sowie der abschließende Text von John Trumbo über „Visual Literacy in Science Communication“ allein schon mit ihren Titeln belegen. Aber statt auf modische Themen und prominente Namen zu setzen, versammelt Pauwels in diesem Rahmen einen vorläufigen Forschungsertrag zur Konstruktion und Funktion von Visualisierungen, denn fast alle der hier wieder abgedruckten Aufsätze sind inzwischen über zehn Jahre alt - wobei nicht alle Studien mit der Alterung gleichermaßen an Gewicht hinzugewonnen haben wie die oben erwähnte von Lynch (der obendrein gleich auch noch mit seinem Überblicksartikel „The Production of Scientific Images" vertreten ist).

Das Programm einer visual literacy ist zugleich Thema einer Reihe von Fallstudien, waren doch naturhistorische und wissenschaftliche Sammlungen und die daraus hervorgegangenen Museen für Wissenschaft und Technik in den Jahrhunderten und Jahrzehnten vor der Erfindung sogenannter Science Centers primär Orte der Veranschaulichung und der Einübung eines wissenden 
Sehens. Im Modus der Vermittlung wissenschaftlichen Wissens ging es dabei zugleich immer auch um eine Inszenierung von gesellschaftlicher Ordnung, wie Sally Gregory Kohlstedt in ihrem Beitrag über Naturhistorische Museen in den USA (in Shteir/Lightman) hervorhebt, oder von geschlechtsspezifischen Hierarchien, wie sie Alan Bewell anhand der Figur des Sammlers (ebd.) analysiert, oder von politisch sanktionierten Visionen vom technischen Fortschritt, wie Eva A. Mayrings Beitrag zur Gemäldesammlung im Deutschen Museum (in Gall) anschaulich belegt. Und selbstverständlich darf unter dem Stichwort Visuelle Allgemeinbildung nicht der Hinweis auf das Projekt von Otto Neurath fehlen, der in seinem Wiener Gesellschafts- und Wirtschaftsmuseum beziehungsweise später in der Emigration mittels der von ihm entwickelten Bildersprache ISOTYPE (eine Art visuelles Esperanto) gerade mit bildlichen Mitteln eine sowohl hinsichtlich ihrer Themen als auch ihres Adressatenkreises wahrhaft allgemeine Bildung realisieren wollte - wie Sybilla Nikolow in ihrem Beitrag zum eigenen Sammelband sowie in Stahnisch/Bauer darlegt. Wie ein solches Projekt heute aussehen könnte, wo Fortschrittsglaube und Einheitswissenschaft weitläufiger Skepsis Platz gemacht haben, darüber reflektiert Jochen Hennig am Beispiel der Erarbeitung einer Konzeption für eine kleine Sonderausstellung zu Atombildern im Deutschen Museum in Gall: Das Potenzial visueller Allgemeinbildung liege heute weniger in der Lesbarkeit der dargestellten Atomphysik, sondern eher darin, die kulturellen Codierungen und gesellschaftlichen Brüche erkennbar werden zu lassen, die noch in scheinbar dokumentarische oder rein wissenschaftliche Bilder eingeschrieben sind.

Hüppauf und Weingart nennen das Durchschauen der Regeln für Medienaufmerksamkeit ein wichtiges Thema einer visuellen Allgemeinbildung und widmen entsprechend eine ganze Sektion den populären Bildern von Wissenschaft in Film und Fernsehen, die von Bruce Clarke mit einem Vorabdruck aus seinem neuen Buch Posthuman Metamorphosis gekrönt wird. Gleichwohl bleibt hier wie in den anderen Sammelbänden ein Gedanke merkwürdig unterbelichtet, den auszuarbeiten sich eigentlich lohnen sollte, nämlich dass die Beschäftigung mit Visualisierungen selbst als eine Signatur des Medienzeitalters zu lesen ist. Wenn digitale Bilder visuelle Realitäten artifizieller Natürlichkeit schaffen, in denen Imitation und Simulation im Nebel elektronischer Bildwelten zusammenfallen, sind Visualisierungen dann nicht die „Mythologie des digitalen Zeitalters“ ${ }^{3}$ ? Es kann doch kein Zufall sein, dass Visualisierung genau in dem Moment massiv zum Anlass und Ausgangspunkt kritischer Reflexion wird, wo digitale Bildwelten so bestechend real geworden sind.

\section{Die Crux mit den Bildern}

Warum bleiben ausgerechnet so viele Bücher zum Thema Visualisierung im Hinblick auf ihre grafische Gestaltung unbefriedigend? Bei Stahnisch/Bauer 
ist das Druckbild viel zu eng, und obendrein erschwert die Verdrängung der Abbildungen in einen Anhang die Lesbarkeit der Beiträge (aber immerhin wurden dadurch Farbabbildungen möglich). Bei Hüppauf/Weingart (2008) sind die Abbildungen in stark schwankender, gelegentlich ungenügender Qualität gedruckt, die nur noch von dem sorglosen Umgang mit Bildern im Sammelband von Elkins unterboten wird. Da drängt sich der Eindruck auf, dass ein Verlagshaus wie Routledge (das beide Sammelbände publiziert hat) sich für das Aussehen seiner Bücher offenbar nicht mehr interessiert. Und man kann Hüppauf und Weingart nur beglückwünschen, für die deutsche Ausgabe mit Transcript einen engagierten Verlag gefunden zu haben.

Positiv hervorzuheben ist hier ebenso der Wallstein Verlag, der den Band von Gall vorzüglich ausgestattet, in Fadenheftung gebunden und mit durchweg sehr guten Abbildungen produziert hat, die immer dann Farbigkeit zeigen, wenn dies für die Analyse oder Erkennbarkeit günstig ist. Dieser Band belegt auch, dass die Sorgfalt bei der Gestaltung der Druckvorlagen viel wichtiger als das Format der schließlich gedruckten Bilder bleibt, denn selbst verhältnismäßig klein gedruckte Abbildungen leisten hier Erstaunliches. Auch Shteir/Lightman ist ein von seinen Bildern her insgesamt überzeugender Band, obwohl ihm Farbabbildungen fehlen. Hier wirkt sich insbesondere das deutlich größere Format des Buches günstig aus. Sollte es forthin für die Reihe verbindlich sein, wäre dem Verlag für den ökonomischen Mut zu danken, denn bedauerlicherweise hat Pauwels in derselben Reihe noch ein etwas kleines Format.

\section{Wissenschaft zu Markte tragen: eine Nachbemerkung}

Die hier vorgestellten Sammelbände regen nebenbei zu soziologischen Beobachtungen über die gegenwärtige Wissenschaftspraxis an: Beinahe alle Bände gehen auf Tagungen zurück, deren Ergebnisse unter den gegenwärtigen Bedingungen effizienzgesteigerter wissenschaftlicher Produktion offenbar keineswegs verloren gehen dürfen, sondern unverzüglich in wissenschaftlichen Output in Form von Publikationen umgewandelt werden müssen.

Aber wie stark unterscheiden sich dabei die akademischen Arbeitsbedingungen auf beiden Seiten des Atlantiks. Während hier Nachwuchswissenschaftlerinnen und Nachwuchswissenschaftler unter Publikationszwang um die wenigen Dauerstellen konkurrieren, erlaubt etwa der kanadische Kontext, in dem der Sammelband von Shteir/Lightman entstanden ist, ein sogenanntes Advanced Research Colloquium, um auswärtige Gäste zur intensiven Diskussion ihrer Studien an die York University einzuladen. Insgesamt sind die englischsprachigen Publikationen fast ausschließlich reserviert für Beiträge von Professorinnen und Professoren auf komfortablen Dauerstellen. Elkins verarbeitet noch die Diskussionsbemerkungen einiger promi- 
nenter Freundinnen und Freunde zur Teilveröffentlichung einer großen Tagung, Pauwels komponiert weitestgehend aus Sekundärveröffentlichungen von Professoren mit Festanstellung einen neuen Sammelband, und auch die deutschen Professoren Hüppauf und Weingart setzen auf vergleichsweise viele international prominente Kolleginnen und Kollegen. Im Vergleich dazu werden deutschsprachige Sammelbände offenbar typischerweise von Nachwuchswissenschaftlerinnen und Nachwuchswissenschaftlern beschickt und herausgegeben. In Stahnisch/Bauer hat nur ein Zweitautor eine Professur, und bei Nikolow/Schirrmacher sind alle Fallstudien aus befristeter Projektarbeit mit Drittmitteln entstanden.

Immerhin sind mittlerweile hüben wie drüben Beiträge von Autorinnen ebenso selbstverständlich wie solche von Autoren, und auch der Bereich von Gender \& Science ist längst keine reine Frauensache mehr, denn mehr als ein Viertel der Beiträge zu Shteir/Lightman wurde von Männern verfasst. Wenn allerdings fast alle Fallstudien im Sammelband von Nikolow und Schirrmacher von Autorinnen auf befristeten Projektstellen stammen, muss das wohl auch als ein Hinweis auf die hierzulande nach wie vor prekäre Stellensituation von Wissenschaftlerinnen gelesen werden.

\section{Anmerkungen}

1 Ordnungen der Sichtbarkeit nannte denn auch Peter Geimer 2002 seinen viel beachteten Sammelband mit einschlägigen Texten zur Geschichte fotografischer Darstellungsverfahren in Wissenschaft und Kunst.

2 Mitchell ist gleich in zwei Sammelbänden vertreten und zeigt sich dabei als Chamäleon. Während er in Weingart/Hüppauf 2008, 2009 den hard scientist mimt, der Neurophysiologie, Kognitionswissenschaft und Wahrnehmungspsychologie zur objektiven Erforschung von Bildwirkungen verknüpfen will, skizziert er in Elkins 2006 eine diskurstheoretisch avancierte picture theory.

3 Das schöne Wort entnehme ich der Einleitung von Hüppauf/Weingart 2008, die es allerdings nur klassisch ideologiekritisch in einem aufklärerischen Sinne verwenden. Aber sind es nicht längst Bildmaschinen, die unsere Seh(n)süchte beflügeln?

Cornelius Borck

Institut für Medizingeschichte und Wissenschaftsforschung

Universität zu Lübeck

Königstraße 42

D-23552 Lübeck

Email: borck@imgwf.uni-luebeck.de 
